\title{
COMPARAZIONE E TRADUZIONE: DALLA LETTERATURA AL DIRITTO
}

COMPARISON AND TRANSLATION:
FROM LITERATURE TO LAW

ALBERTO VesPAZIANI

Professore Associato confermato di Diritto Pubblico Comparato presso l'Università del Molise, dove ha insegnato anche Diritto e Letteratura, Diritti fondamentali europei, Istituzioni di diritto pubblico, Diritto dell'ambiente, Diritto dell'Unione europea e Teoria della comunicazione e dell'argomentazione. Molise, Itália. alberto.vespaziani@unimol.it

\begin{abstract}
RIASSUNTO
Il contributo discute l'ascesa degli studi sulla traduzione nel diritto comparato. La prima parte riassume $i$ testi letterari classici che costituiscono le fonti di ispirazione per l'analisi giuridica contemporanea sulla traduzione, la seconda parte analizza i principali argomenti addotti da Ost nel suo libro Traduire, la terza parte avanza alcune proposte di ricerca per le future conessioni tra comparazione e traduzione nell'analisi giuridica europea. Il contributo sostiene che la svolta verso la traduzione dovrebbe essere accolto dai giuristi come una sfida per il perseguimento di una cultura costituzionale comune.
\end{abstract}

Parole chiave: comparazione; traduzione

\begin{abstract}
The paper deals with the rise of translation studies in comparative law. The first part summarizes the classical literary texts which constitute the sources of inspiration for contemporary legal analysis on translation, the second part reviews the main arguments advanced by Ost in his book Traduire, the third part sketches some research proposals for the future implications between comparison and translation in European legal analysis. The paper argues that the translation turn should be welcomed by European jurists as a challenge for the pursuit of a common constitutional culture
\end{abstract}

Keywords: comparison; translation 


\section{SOMMARIO}

INTRODUZIONE; 1 I TESTI CLASSICI SULLA TRADUZIONE: DA JAMES BOYD WHITE A EMILIO BETTI, PASSANDO PER BENJAMIN, STEINER, ORTEGA Y GASSET E RICOEUR; 2 LA TRADUZIONE COME NUOVO PARADIGMA GIURIDICO IN FRANÇOIS OST; 3 PER UNA DOTTRINA (TRADUTTOLOGICA) COSTITUZIONALE COMUNE EUROPEA; RIFERIMENTI BIBLIOGRAFICI.

\section{INTRODUZIONE}

In ambito letterario, e specialmente negli studi di letterature comparate, il tema della traduzione è di casa. In ambito giuridico, viceversa, la traduzione guadagna terreno solo da tempi recenti. Negli studi contemporanei di diritto comparato, in particolare, la traduttologia si afferma come una nuova frontiera e talvolta persino come autonoma disciplina. In questo contributo mi propongo di evidenziare il nesso crescente, all'interno degli studi giuridici, tra comparazione e traduzione. Lo scritto si divide in tre parti: nella prima parte offro una ricognizione dei testi letterari classici che hanno tematizzato la traduzione; nella seconda parte riassumo alcuni argomenti avanzati da un maestro europei degli studi di diritto e letteratura, François Ost, nel suo libro Traduire; nella terza parte indico alcune possibili linee di sviluppo traduttologiche per una dottrina costituzionale comune europea.

\section{I TESTI CLASSICI SULLA TRADUZIONE: DA JAMES BOYD WHITE A EMILIO BETTI, PASSANDO PER BENJAMIN, STEINER, ORTEGA Y GASSET E RICOEUR}

Sei anni fa l’Accademia nazionale dei Lincei, una delle più prestigiose istituzioni culturali italiane, ha dedicato un convegno internazionale al tema della traduzione giuridica. I contributi insistevano sul carattere pionieristico degli studi traduttologici in campo giuridico; in effetti la tematizzazione della traduzione è piuttosto recente nel campo degli studi giuridici. In Italia il maestro del diritto privato comparato Rodolfo Sacco è stato forse il primo a dedicarvi una speciale attenzione, notando che «attualmente si ha l'impressione che nei prossimi venti anni i problemi di traduzione diventeranno il capitolo più promettente della comparazione giuridica e che apriranno vie importantissime all'epistemologia giuridica e alla riforma della lingua giuridica» (Sacco 2000, 723). Sulla scia di Sacco la dottrina privato-comparatistica più accorta (Pozzo 2008) ha lavorato sul nesso che intercorre tra diritto dell'Unione europea e diritto comparato, proprio in virtù della natura multilinguistica del diritto comunitario, che si 
fonda sull'assenza di un'unica lingua ufficiale. Significativamente, in questo lavoro pionieristico i privatisti si sono messi in luce più dei pubblicisti, concentrandosi tuttavia solo sugli aspetti pratici della traduzione, finendo per offrire una sofisticatissima tecnologia traduttologica. Viceversa, forse per il retaggio del nazionalismo metodologico in cui la dottrina dello stato aveva confinato gli studi del diritto costituzionale, i comparatisti del diritto pubblico sembrano essersi spostati dallo studio della costituzione nazionale allo studio del diritto comunitario o globale, senza tuttavia passare per lo studio della traduzione giuridica. Per iniziare ad individuare delle possibili linee di sviluppo traduttologiche per la dottrina costituzionale comune europea, passo quindi ad individuare i testi classici e le fonti di ispirazione per la ricerca sull'intreccio tra costituzione, comparazione e traduzione.

Fortunatamente proprio il capofila del movimento statunitense Law \& Literature, James Boyd White, ha dedicato al tema della traduzione una delle sue maggiori opere, concepita come sequel al volume When Words Lose Their Meaning, la cui traduzione italiana ad opera di Barbara Pozzo viene presentata proprio in questo convegno. Boyd White sostiene quattro tesi fondamentali sulla traduzione: «(1) essa è un'arte del riconoscimento e della risposta, tanto nei confronti di un'altra persona quanto rispetto ad un'altra lingua; (2) essa conduce il traduttore ad un punto tra i linguaggi, tra la persone (e tra i popoli), dove le differenze reciproche possono essere viste meglio e comprese più da vicino - differenze che ci permettono di vedere in una nuova maniera ciò che ognuno è, o, più precisamente, differenze in cui risiedono il significato e l'identità di ognuno»; (3) essa implica un'affermazione del sé, nonché del proprio linguaggio, che è anche simultaneamente una limitazione di entrambi; e (4) che essa è in tutti questi rispetti un modello del diritto e della giustizia, giacché questi due sono in essenza dei modi di stabilire giuste relazioni, sia tra una persona ed un'altra, sia tra una mente e il linguaggio che incontra» (Boyd White, 1994, 231). La riflessione di White si snoda lungo i sentieri della traduzione letteraria, della teoria economica, dell'argomentazione giudiziale, per giungere alla giurisprudenza costituzionale ed infine alla teoria morale. White sottolinea come la traduzione ha tanto una dimensione intellettuale quanto una dimensione etica: essa «riconosce l'altro - il compositore del testo originale - come un centro di significato separato dal sé. Essa richiede che si scoprano tanto il valore del linguaggio dell'altro quanto i limiti del proprio. La buona traduzione non procede secondo i motivi del dominio o dell'acquisizione, ma secondo il rispetto. Essa è una parola che indica un insieme di pratiche attraverso cui impariamo a vivere con la differenza, con la fluidità della cultura e con l'instabilità del sé. Essa non è un'operazione 
intellettuale sulla materialità, ma un modo di essere se stessi in relazione ad un altro essere» (Boyd White, 1994, 257).

Se dunque il tema della traduzione è stato già analizzato dalla prospettiva Law \& Literature statunitense, è proprio nel campo degli studi letterari che si trovano i testi classici sulla traduzione. Su questo tema, senza dubbio, il testo più citato è quello di Walter Benjamin, Il compito del traduttore. E' bene ricordare che già nella traduzione del titolo del saggio si annida una ricchezza polisemica: die Aufgabe vuol dire infatti tanto il compito assegnato, lo scopo da conseguire, quanto l'abbandono, la rinuncia al perseguimento dello scopo assegnato, che si rivela irraggiungibile. In effetti la riflessione di Benjamin si articola tutta sull'idea regolativa di una lingua pura, che orienta ogni tentativo di traduzione: «il grande motivo dell'integrazione delle molte lingue nella sola lingua vera è quello che ispira il suo lavoro. Un lavoro in cui le singole proposizioni, opere, giudizi, non giungono mai ad intendersi - come quelli che restano affidati alla traduzione -, ma in cui le lingue stesse concordano fra loro, integrate e riconciliate nel modo del loro intendere. Ma se c'è una lingua della verità, in cui gli ultimi segreti intorno a cui ogni pensiero si affatica sono conservati senza tensione e quasi tacitamente - questa lingua della verità è la vera lingua. E proprio questa lingua, nel presentire e descrivere la quale è la sola perfezione cui il filosofo può aspirare, è intensivamente nascosta nelle traduzioni. Non c'è una musa della filosofia, e non c'è nemmeno una musa della traduzione» (Benjamin 1962, 47).

Anche George Steiner ha messo la traduzione al centro delle sue riflessioni in Dopo Babele. Per Steiner la traduzione è formalmente e pragmaticamente implicita in ogni atto di comunicazione, nell'emissione e nella ricezione di ogni modalità di significato, sia nel senso semiotico più ampio, sia negli scambi verbali specifici. Capire vuol dire decifrare. Ascoltare il significato vuol dire tradurre. Quindi la struttura essenziale, i mezzi esecutivi ed i problemi dell'atto della traduzione sono interamente presenti negli atti del discorso, della scrittura, della pittura all'interno di ogni linguaggio. La traduzione tra diversi linguaggi è un'applicazione particolare di una configurazione e di un modello fondamentale del linguaggio umano persino quando esso è monoglotta (Steiner, 1994, xii). La traduzione è quindi innanzitutto interna a ciascuna lingua; la pluralità linguistica rappresentata dal mito di Babele è dunque tanto una maledizione, perché genera equivoci e conflitti, tanto una benedizione, un dis-astro, una pioggia di stelle di significati sull'uomo.

Quest'ambivalenza della traduzione è anche sottolineata da Ortega y Gasset, che in una serie di articoli pubblicati su La Nación di Buenos Aires nel 1937 riflette sull'intraducibilità di 
ogni testo. In fondo, così Ortega, la traduzione è un utopia, qualcosa di impossibile e di irrealizzabile. E tuttavia sempre si è tradotto e sempre si tradurrà. Ortega chiama l'attività del tradurre faena, vale a dire un'impresa, una fatica. La traduzione esprime sia il testo originario, sia i limiti delle lingue implicate. Nella traduzione sono i termini intraducibili ad essere più interessanti: «non si capisce nella sua radice la stupenda realtà che è il linguaggio se non si comincia dall'avvertire che il parlare si compone soprattutto di silenzi. Un essere che non fosse capace di rinunciare a dire molte cose, sarebbe incapace di parlare. E ogni lingua è un'equazione differente fra manifestazioni e silenzi. Ogni popolo tace alcune cose per poterne dire altre. Perché tutto sarebbe indicibile. Da qui deriva l'enorme difficoltà della traduzione: in essa si tratta di dire, in un idioma, proprio le cose che questo idioma tende a silenziare. Ma nel contempo, si intravede ciò che il tradurre può avere di magnifica impresa: la rivelazione dei segreti reciproci che i popoli e le epoche si celano a vicenda e che tanto contribuiscono alla loro dispersione e ostilità; insomma, un audace integrazione dell'Umanità. Perché come diceva Goethe: 'solo dall'insieme di tutti gli uomini l'umano è vissuto completamente'» (Ortega y Gasset 2001, 43).

Anche Paul Ricoeur condivide la necessità di non confinare la traduzione dentro il recinto della linguistica, ma di ricomprenderla dentro un orizzonte ermeneutico, che renda giustizia alla storicità dell'uomo ed al suo desiderio di giustizia. Per Ricoeur il mito di Babele significa tanto dispersione geografica quanto confusione sul piano della comunicazione. E tuttavia, così Ricoeur, è necessario uscire tanto dall'alternativa teorica - traducibile versus intraducibile -, quanto dall'alternativa pratica - fedeltà versus tradimento, salvo ammettere che la pratica della traduzione resta un'operazione a rischio, sempre alla ricerca della sua teoria. Per Ricoeur la traduzione, sia nel versante extra-linguistico sia nel versante intra-linguistico, si rivela teoricamente incomprensibile, ma effettivamente praticabile. In questo senso l'orizzonte messianico della lingua pura, come evocato da Benjamin, non aiuta l'attività del traduttore: «purtroppo la pratica della traduzione non riceve alcun soccorso da questa nostalgia tramutata in attesa escatologica; e forse bisognerà affrettarsi a rinunciare al desiderio di perfezione, per assumere senza ebbrezza e con estrema sobrietà il 'compito del traduttore' (Ricoeur 2001, 57). Tesa tra la dispersione multilinguistica e l'aspirazione alla lingua unica, la traduzione si configura come un'attiva pratica, non compiutamente teorizzabile, e tuttavia eticamente necessaria: «questo è il bilancio sommario della battaglia che oppone il relativismo empirico, che conclude per l'impossibilità della traduzione, e il formalismo accademico, che fallisce nel fondare il dato della traduzione su una struttura universale dimostrabile. Sì, bisogna 
ammetterlo: da una lingua all'altra, la situazione è proprio quella della dispersione e della confusione. E tuttavia la traduzione si inscrive nella lunga litania dei 'nonostante tutto'. A dispetto dei fratricidi, noi militiamo per la fraternità universale. A dispetto dell'eterogeneità degli idiomi, esistono bilingui, poliglotti, interpreti e traduttori» (Ricoeur 2001, 59). Per Ricoeur, dunque, la traduzione è di fatto, non di diritto. «La pratica della traduzione e l'asserzione della traducibilità hanno una funzione anche, e soprattutto, etica. Esse rappresentano, in primo luogo, la condizione inevitabilmente plurale dell'umanità. Così come il linguaggio esiste solamente nelle lingue, l'umanità esiste solamente nella frammentazione degli uomini» (Goldoni 2007, 252).

In particolare l'invito di Ricoeur a non attendere l'arrivo del traduttore perfetto, ma a dedicarsi prosaicamente al compito di traduzione quotidiano, echeggia l'invito con cui Max Weber aveva concluso la sua famosa conferenza sulla Scienza come professione: «La situazione attuale di tutti coloro i quali vivono nell'attesa di nuovi profeti e nuovi redentori è la medesima che viene descritta nel bellissimo canto della sentinella di Edom, poi raccolto tra gli oracoli di Isaia: 'Mi gridano da Seir: 'Sentinella, quanto durerà ancora la notte?' La sentinella risponde: 'Verrà il mattino, ma è ancora notte. Se volete domandare, tornate un'altra volta". Il popolo a cui fu data questa risposta ha domandato e atteso per ben più di duemila anni, e ne conosciamo il triste destino. Morale: desiderare e attendere non basta. Noi faremo diversamente, ci metteremo al lavoro e adempiremo al compito quotidiano sia come uomini, sia come professionisti. E' una cosa facile e immediata - quando ognuno abbia trovato e dato ascolto al demone che tiene i fili della sua vita» (Weber 1997, 133).

E' proprio raccogliendo i fili della propria vita, che Derrida ha offerto una meditazione sulla decostruzione della traduzione. Per Derrida, infatti, ogni individuo non parla che una sola lingua, e tuttavia questa non è la sua (Derrida 2004, 5). Da una parte il linguaggio non appartiene totalmente all'uomo, che non lo può interamente possedere, dall'altra ogni lingua ospita al suo interno altre lingue. La decostruzione della traduzione non si limita a registrare il fatto della pluralità delle lingue, ma evoca il silenzio che attraversa ogni lingua, come ciò che non può esserne contenuto, e così la interrompe, le impedisce di chiudersi, di irrigidirsi in un discorso totalitario. La traduzione mostra la condizione paradossale del soggetto parlante: da una parte non si parla mai che una sola lingua - o piuttosto un solo idioma, ma dall'altra parte non si parla mai una sola lingua - o piuttosto non c'è idioma puro. Seguendo questa prospettiva «si tratta sempre di inventare. Di innovare la propria lingua, di ritradurla, per non cancellare in essa quella dimensione di ospitalità che si apre nella sua inadeguatezza, nelle ambiguità, nei 
lunghi giri e deviazioni che è costretta a percorrere, senza potersi mai acquietare in un punto d'arrivo stabile e condiviso, senza potersi mai appoggiare su un senso che la preceda» (Berto 2004, XX).

L'appello all'umanità comune come presupposto della traduzione era stato già invocato da un altro grande esponente della tradizione ermeneutica, Emilio Betti: «la parola, a qualunque lingua appartenga fa pur sempre appello alla comune umanità, nella quale tutti gli spiriti, oltre il particolare orizzonte di ciascuno, si sentono affini e pertanto in grado di intendersi: questo è il comune presupposto di ogni traduzione» (Betti 1990, 656).

Nella sua Teoria generale dell'interpretazione Betti articola due sensi della traduzione: in un senso generale «ove si consideri come fatto spirituale, non c'è interpretare che non sia un ricostruire, ossia un riprodurre interiormente, un perenne tradurre dall'altrui idioma e linguaggio nel proprio» (Betti 1990, 636). In un senso più specifico, invece, Betti colloca la traduzione all'interno della categoria dell'interpretazione riproduttiva: «il problema specifico del tradurre sta nel sostituire ad una forma rappresentativa non intelligibile un'altra forma equivalente che riesca intelligibile ad una cerchia di lettori (o di ascoltatori) diversa da quella cui era rivolta la prima. Si tratta di sostituire, cioè di assorbire e soppiantare (nel senso del ted. 'aufheben') la forma originale, non già di giustapporvi un'altra forma, che ad essa rinvii, la chiarisca e la lumeggi (finalità, questa, del commento), o che, a guisa d'incitamento, serva soltanto a spronare i lettori ben disposti a cimentarsi direttamente con l'originale. Certo la traduzione può anche servire d'incitamento e di rinvio - come accade colà dove essa non basti da sola a procacciare una piena intelligenza del testo tradotto -; ma la sua funzione propria è sostitutiva» (Betti 1990, 660).

Per Betti dunque la traduzione è sia una figura specifica dell'interpretazione riproduttiva - una riproduzione dell'originale che rimane fedele all'idea, al senso, al pensiero -, sia una componente essenziale di ogni attività interpretativa. Di recente, questo nesso tra traduzione e interpretazione è stato rivisitato da uno dei maestri del movimento Droit et literature europeo: François Ost.

\section{LA TRADUZIONE COME NUOVO PARADIGMA GIURIDICO IN FRANÇOIS OST}

La lettura ermeneutica della condizione multilingue in cui si viene a trovare il diritto contemporaneo europeo conduce Ost ad individuare nella categoria della traduzione il tema 
centrale delle riflessioni comparatistiche. Da tempo sostenitore del tramonto delle concezioni piramidali dell'ordinamento giuridico, per Ost la traduzione opera all'interno dell'ordinamento giuridico reticolare europeo. La rete di comunicazione rappresenta un cambio di paradigma nelle scienze sociali; nel campo della linguistica, ad esempio, si assiste ad una rivoluzione copernicana e ad una flessione del paradigma comunicativo a favore del paradigma della traduzione. Con il suo libro Traduire, Ost intende contribuire all'affermazione e all'approfondimento del paradigma traduttivo. Ma in ragione della forza d'inerzia del modello comunicativo dominante, del suo radicamento nei fantasmi babelici, e del suo legame con potenti interessi economici (il linguaggio unico della pubblicità e della propaganda), la promozione del multilinguismo e della traduzione risvegliano spesso un atteggiamento polemico, come spesso accade nelle situazioni di lotta tra paradigmi rivali. Entrano in gioco una serie di verità paradossali che sono come il filo rosso della sua opera: 1) l'episodio di Babele racconta un lieto evento: la molteplicità delle lingue è una benedizione; 2 ) le lingue naturali sono assai più universali che le cosiddette "lingue perfette" che pretendono di abolire Babele; 3 ) la traduzione opera soprattutto all'interno di ciascuna lingua, prima di operare alle loro frontiere; 4) l'intraducibilità è la condizione di possibilità della traduzione e non la ragione del suo scacco; parimenti si compara veramente solo ciò che è incomparabile; 5) la traduzione è compiutamente scrittura, perché l'originale è sempre, più o meno, di seconda mano; 6) Non si parla necessariamente per comunicare, ancor meno per comunicare delle informazioni veritiere; 7) Se è vero che bisogna rassegnarsi all'impossibilità della traduzione perfetta, allora la creatività è il segno delle traduzioni fedeli; 8) "la lingua dell'Europa è la traduzione"; il multilinguismo è un vantaggio e non un ostacolo per l'Europa; 9) La traduzione dà accesso, sul piano etico, al "sé come un altro", piuttosto che all'altro come un alter ego, preservandosi così dalle sue derive assimilatrici ed egemoniche (Ost 2009, 12).

E' stupefacente constatare che $i$ due miti della lingua e della legge perfetta sono sistematicamente ravvivati nei periodi di grande tensione sociale: divisioni politiche, guerre di religione, difficoltà economiche. Ogni volta che l'Europa cede ai suoi vecchi demoni e minaccia di lacerarsi, si ergono delle personalità straordinarie, da Raimondo Lullo a Leibniz, da Pico della Mirandola a Bentham, per rianimare la fiamma di una lingua o di una legge universale (Ost 2009, 69).

Così vanno le lingue dopo Babele: oscillanti incessantemente tra la celebrazione del genio dei discorsi indigeni e della loro brulicante molteplicità, e la nostalgia segreta di un 
parlare luminoso, Pentecoste trionfante, glossolalia finale che, alla maniera del linguaggio della musica, riunirà tutti gli uomini in una comune cattura del mondo.

Anche sul versante giuridico lo sviluppo ipertrofico dei diritti nazionali non ha totalmente soffocato, nel XX secolo, la ricerca della legge perfetta. Istruita dai fallimenti della concezione di un diritto razionale a priori, questa ricerca intraprende oggi delle strade più indirette. E quindi si procederà anche a posteriori, sulla base delle esperienze acquisite e di una diversità giuridica che è oramai non riducibile. Una prima via, modesta e sicuramente indiretta, consiste nello sviluppo degli studi di diritto comparato. Si raccoglie, si classifica, si compara. Pratica ambivalente, tuttavia, che valorizza contemporaneamente la diversità e le specificità, e nondimeno non si distacca completamente dal sogno segreto di far emergere delle strutture comuni, delle logiche trasversali, delle costanti. E quando il contesto politico si presta, così Ost, si arrischierà persino a schizzare delle soluzioni comuni - delle soluzioni non imposte a priori, ma risultate a posteriori dalla comparazione. E' sufficiente evocare, a titolo di esempio riuscito di questo modo di procedere, l'unificazione di certi settori del diritto materiale nord-americano attraverso la legislazione federale e un certo numero di convenzioni di unificazione o di armonizzazione del diritto materiale adottate nella conferenza dell'Aia dei diritto internazionale privato, dell'Istituto internazionale per l'unificazione del diritto privato.

Una seconda via, più tradizionale, che prende a prestito oggi la ricerca di un diritto comune, è l'elaborazione di un diritto internazionale sempre più integrato e universale (Ost 2009, 87).

La lingua pura vagheggiata da Benjamin non è una lingua perfetta, anzi non è neanche una lingua, ma soltanto un postulato della traduzione, un'idea regolatrice, una terra promessa (senza dubbio mai raggiunta). La traduzione occupa il posto lasciato vacante da tutti i progetti abortiti di abolizione di Babele (Ost 2009, 104).

Da una rassegna storica Ost rileva la diversità pressoché infinita delle figure della traduzione. La Grecia, fieramente monolingue, non traduce. La cultura romana esiste solo grazie alla traduzione; una traduzione egemonica e platonizzante. Quando si annette la cultura dell'altro si crede di poterne appropriare il senso (lure victoris per privilegio del conquistatore) facendo economia della sua forma. Nell'universo cristiano la traduzione si fa militante ed universalista: sulle rovine di Babele si tratta di proclamare in tutte le lingue la buona novella della salvezza del mondo. Ma quando si ha a che fare con dei testi sacri, la traduzione torna, presso Girolamo e Lutero, ad un rispetto maggiore per la lettera. Con la modernità individualista e nazionalista, la diversità delle lingue viene valorizzata in quanto tale, ed ognuna compete 
nella pretesa di dire l'universale. In questo gioco la Francia spicca, in nome del suo "genio" nazionale, con la traduzione libera dei Romani. Ma la Germania romantica le succede, declinando la traduzione in un modo cosmopolitico; la crescita di ogni cultura (Bildung) passa per la mediazione delle opere straniere. Quanto al mondo contemporaneo globalizzato, esso pratica la traduzione in un modo industriale: su grande scala, ma col rischio della standardizzazione, come se si trattasse soltanto di uno scambio di informazioni (Ost 2009, 124).

Allora traducibile o intraducibile? Humboldt consacra quindici anni della sua vita a tradurre l'Agamennone di Eschilo. Ciò non gli impedisce di iniziare la sua prefazione con le parole "un tal poema è intraducibile (unübersetzbar)".. e di dichiarare: "ciò non deve dissuaderci dal tradurre”. In queste condizioni l'intraducibile è, come dice Cassin, ciò che non cessa di (non) tradurre. Seguendo l'esempio della catastrofe di Babele, l'intraducibile è l'occasione e non la maledizione della traduzione. È il segno che nel discorso qualcosa "resiste" e dunque innova; annuncia una parola, e non soltanto la comunicazione di un'informazione. “Nella traduzione si deve arrivare sino all'intraducibile" scriveva Goethe. Ancor meglio: in un certo modo la traduzione non comincia che con la coscienza dell'intraducibile; prima di ciò non c'è che trasposizione spontanea, sostituzione non problematica, svolgimento del circolo ermeneutico della connivenza linguistica e culturale. Da questo punto di vista Ost può sostenere, senza provocazione, che l'intraducibile è la condizione di possibilità della traduzione; ma anche la sua condizione d'impossibilità, e dunque l'assicurazione del suo scacco - ciò che, a ben vedere, è la miglior garanzia della sua riuscita. Giacché, se la traduzione dovesse riuscire totalmente, lo spettro della lingua perfetta si riaffaccerebbe in superficie, e le torri ricomincerebbero a vacillare.. (Ost 2009, 177).

Ost avanza un'etica della traduzione, non una sua morale. L'etica, con la sua prospettiva teleologica basata sui valori, precede ed ingloba la morale, il cui punto di vista è deontologico e si traduce in norme. Occorre dunque riflettere in termini di fondazione etica e non di eventuali prescrizioni normative. Qual è dunque lo scopo della prospettiva traduttrice? In una parola: liberare una terza via , quella di uno spazio condiviso tra il linguaggio (e il pensiero) unico da una parte - l'esperanto o il globish o il globais, per esempio -, ed il ripiegamento sugli idiomi singoli dall'altro. Tra la Cariddi dell'onnitraducibilità proclamata da un linguaggio dominante che crede di poter assorbire tutto nella sua ipseità, e la Scilla dell'ombroso intraducibile dietro cui si rifugiano delle lingue (culture, comunità) gelose delle loro specificità, la traduzione mira a tracciare un percorso. Ost ricorda che nella settima città non si tratta solamente di garantire la traduzione delle lingue in senso stretto; si tratta piuttosto di inventare 
delle modalità di traduzione all'interfaccia delle scienze, delle culture, delle tradizioni, dei costumi, dei codici giuridici prevalenti nelle molteplici "sfere della giustizia" nel senso di Walzer. Questa riflessione apre dunque, sul piano della filosofia politica, la possibilità di pensare una terza via, così spesso inseguita, tra liberalismo politico e comunitarismo; sul piano della filosofia del linguaggio essa potrebbe contribuire ad articolare la concezione formale della lingua come lessico, con la prospettiva più sostanziale che la concepisce come un'enciclopedia (290).

Al cospetto delle ricorrenti strategie riduzionistiche, che mirano all'uniformità del linguaggio unico, le scienze umane e sociali progrediscono soltanto grazie alle virtù della comparazione: non solo la semplice giustapposizione delle varietà alla maniera di una classificazione botanica, ma una comparazione che conduca ai concetto, metodi e teorie più che agli oggetti, e tutto ciò all'interno di una prospettiva critica di reciproca messa in discussione. Al cuore del comparativismo critico ritroviamo la traduzione all'opera. Una traduzione dedicata tanto alla messa in equivalenza di ciò che è comparabile, quanto all'identificazione dei punti di resistenza, laddove le rappresentazioni e i concetti cessano di coincidere. $\dot{E}$ in queste zone di frattura, in queste faglie dove il pensiero conosce il suo massimo movimento, che la ricerca ha le più grandi chances di progredire. La traduzione rivela qui pienamente le sue virtù euristiche: nello smascherare le approssimazioni, i clichés, i luoghi comuni e altre pigrizie del pensiero, essa offre un'occasione unica di approfondire la sua comprensione. Problematizzando ciò che sembrava essere evidente, essa si rivela essere una scuola inimitabile di rigore e di creatività. Bisogna avere avuto la fortuna di essere tradotti per sapere che le sfide più serie del pensiero vengono dai colleghi traduttori, questi "amici intrattabili" che, non dandovi nulla, fanno passare i vostri testi su altre rive. Lungi dunque dall'essere un ostacolo alla marcia della scoperta scientifica, la pluralità delle lingue è al contrario la sua sorte: essa preserva la possibilità di formulare delle nuove metafore e di scrivere dei nuovi racconti. Al contrario, plasmare il proprio pensiero nella forma anestetizzata dell'inglese congressuale, vuol dire rischiare di privarsi del punto di vista più originale del proprio discorso; è anche, spesso, il modo per incamminarsi sulla strada di una formalizzazione (pseudo-)matematica della teoria che, se presenta il merito di una traducibilità incontestabile, potrebbe tuttavia conseguire tale risultato al prezzo di una banalizzazione del proposito. L'Europa pensa in più lingue: bisogna sostenere la traduzione più di quanto non sia stato fatto sinora; è un problema di sovvenzioni, ma anche di formazione e di considerazione dei traduttori. Come ha scritto Nies, "la traduzione è il nocciolo della storia intellettuale e dell'identità europea" (Ost 2009, 375). 
Per Ost la traduzione è dunque un paradigma, nel senso di Kuhn. Se è vero che la società contemporanea, diversificata e aggrovigliata, prende oggi la forma della rete, rimane da determinare quale sia la grammatica di questa rete, la sua legge di funzionamento. Spesso vi si risponde mediante le teorie della comunicazione, ivi compresa l'"etica comunicativa" di Habermas. Così si rende giustizia alla "svolta linguistica" che ha, verso la meta del XX secolo, conferito preminenza al problema del linguaggio (affrontato da un angolo essenzialmente pragmatico) nella spiegazione delle interazioni umane. "Rete" e "svolta linguistica" formano così il terreno ideale per un paradigma comunicativo generalizzato. Ma la comunicazione non si lascia ridurre ad un modello semplice di trasmissione-ricezione di un messaggio "ricevuto 5 su 5". Tutta una serie di obliquità sono riuscite ad orientare il messaggio in un senso inatteso; non c'è elemento dello schema che non susciti una serie di problemi - delle "anomalie" riguardo al paradigma comunicativo. Si capisce allora, nel senso di una rivoluzione copernicana, che tra messaggio emesso e messaggio ricevuto, tra intenzione dell'emittente e comprensione del ricevente, si possono scavare degli abissi. A quel punto è scoccata l'ora della traduzione. Ost nota come la comunità dei ricercatori delle scienze sociali fa un uso sempre più frequente del modello traduttivo. La traduzione è dunque un paradigma scientifico che si avvale di metafore, racconti esemplari, leggi fondamentali, visioni del mondo e valori. Proprio il discorso intorno ai valori richiede un'etica della traduzione, e al dilemma eterno tra fedeltà e libertà, Ost risponde in termini di responsabilità: la buona traduzione è una traduzione responsabile in un doppio senso; essa risponde al testo e risponde all'autore - ciò non significa restituirgli la "copia conforme" del suo messaggio, ma di assumerlo un prima persona nella propria lingua. La traduzione, così Ost, non si lascia incasellare negli schemi dei modelli dominanti della comunicazione, dell'argomentazione o della negoziazione, perché questi sono modelli che si ispirano ad una concezione formale e cognitivista della ragione, e dunque sottovalutano tanto il radicamento comunitario delle pretese morali quanto l'ineguale attitudine dei protagonisti a padroneggiare lo scambio discorsivo. Infatti l'etica della discussione funziona nell'orizzonte di un'utopia della parola condivisa, la nuova retorica si appoggia sul presupposto di un uditorio universale che seleziona gli argomenti razionali, la negoziazione si inscrive nella finzione di una concorrenza quasi perfetta e di un'informazione egualmente distribuita... Si tratta tanto di idealizzazione contro-fattuali quanto di versioni di un universalismo troppo precoce che non manca di esercitare tutta una serie di violenze sugli interlocutori (Ost 2009, 386).

Traduire si annuncia come un testo classico, una lettura obbligata per tutti quanti hanno a cuore lo sviluppo del movimento europeo “diritto e letteratura”. Particolarmente 
feconda è la direzione dell'argomentazione di Ost: dall'interpretazione del mito di Babele all'identificazione dei modelli storici delle traduzioni, dalla fenomenologia delle definizioni e delle confutazioni delle obiezioni allo sviluppo delle implicazioni metodologiche, etiche e politiche dell'affermazione della traduzione come paradigma. Dalla letteratura al diritto: per un approccio Diritto e letteratura qui è Rodi ed è qui che occorre saltare. Passo quindi ad accennare alcune possibili linee di sviluppo in cui la dottrina costituzionale europea può mettere a frutto la fecondazione reciproca tra comparazione e traduzione.

\section{PER UNA DOTTRINA (TRADUTTOLOGICA) COSTITUZIONALE COMUNE EUROPEA}

L'eclissi del federalismo politico europeo, e l'aborto del trattato costituzionale, sconsigliano la costruzione dogmatica di un ordinamento costituzionale europeo di tipo unitario. Se è dunque impossibile parlare di un diritto costituzionale europeo unico, è però possibile rintracciare alcune tendenze comuni della dottrina costituzionale europea. La rete europea di costituzionalità (Pernice, 2000) si alimenta del pluralismo culturale delle differenziate sfere pubbliche nazionali e dei relativi, interconnessi ordinamenti giuridici. Per una prospettiva traduttologica non si tratta solo di individuare tecniche di traduzione, ma soprattutto di individuare $\mathrm{i}$ limiti di traducibilità che emergono proprio dalle differenze culturali. Gli intraducibili, già indagati in filosofia, possono anche essere ricercati con le lenti del costituzionalismo comparativo. Quali differenze emergono dalla mancata traduzione di termini quali acquis communautaire, common law, rule of law, governance, fueros, Öffentlichkeit? Perché nel linguaggio costituzionalistico statunitense doctrine viene tradotto in italiano con “indirizzo giurisprudenziale della corte", mentre jurisprudence esprime la riflessione teoretica sul diritto? E quali differenze emergono dal fatto che alcuni lemmi esprimono differenze istituzionali pur rimanendo invariati? Il Bundesrat tedesco è diverso da quello austriaco, che è a sua volta diverso da quello svizzero. Una casistica traduttologica può dunque essere utile alla ricerca delle implicazioni normative della comparazione, ma ciò non deve indurre alla tentazione di edificare una Grand theory traduttologica; alla tentazione ricorrente di costruire una nuova torre babelica capace di chiarificare gli enigmi del diritto globale, occorre ripetere che la traduzione perfetta è teoricamente impossibile, eppure tradurre è una pratica effettiva e necessaria. Più feconda appare viceversa la prospettiva casistica che si concentri sul concreto 
operare delle giurisprudenze europee, che operano traduzioni ed interpretazioni dei diritti fondamentali (Vespaziani 2009, VIII; 2008).

Lo stesso Ost ha notato come i diritti fondamentali si sono elevati in Europa al rango di principi generali che ispirano interi settori della regolazione positiva. Si elabora un diritto comune, nel triplo senso di un diritto accessibile a tutti (nella forma dei ricorsi individuali), del diritto federante le soluzioni disperse nelle varie branche del diritto, e del diritto condiviso dai diversi paesi europei (Ost 2009, 88).

Ecco allora che al cospetto dell'eclissi del federalismo politico si assiste ad una crescita del federalismo dei diritti fondamentali europei, cui corrisponde un turn to translation della dottrina costituzionale, comparativa in quanto traduttologica. D'altronde la crisi della dogmatica statualistica è la crisi delle gabbie concettuali in cui l'imperiale nazionalismo metodologico aveva preteso di rinchiudere il nomos paideico delle narrazioni delle lotte per il riconoscimento di pretese giuridiche fondamentali. La società aperta degli interpreti della costituzione europea può dunque oggi venir alimentata da una dottrina costituzionale comparativa che aspiri a traduzioni costituzionali comuni e all'edificazione di una società aperta dei traduttori della costituzione europea. Certo il compito del traduttore consiste anche nell'abbandono delle illusioni totalizzanti dell'edificazione di sistemi chiusi, compatti e rassicuranti. E tuttavia non attenderemo l'arrivo di una traduzione perfetta della costituzione europea ma, come intellettuali e professionisti, ci dedicheremo con sobrietà e modestia alle nostre traduzioni quotidiane, non per uscire dai nostri orizzonti culturali, ma per divenire consapevoli dei valori che le nostri categorie giuridiche veicolano.

\section{RIFERIMENTI BIBLIOGRAFICI}

ACCADEMIA NAZIONALE DEI LINCEI. Le nuove ambizioni del sapere del giurista: antropologia giuridica e traduttologia giuridica, Roma: Accademia Nazionale dei Lincei, 2010.

BENJAMIN, Walter. Il compito del traduttore. Angelus Novus, Torino: Einaudi, 1962.

BERTO, Graziella. Il disagio della traduzione. In: DERRIDA, Jacques. Il monolinguismo dell'altro, Milano: Raffaello Cortina, 2004.

BETTI, Emilio. Teoria generale dell'interpretazione. Milano: Giuffré, 1990.

BOYD WHITE, James. Justice as Translation. An Essay in Cultural and Legal Criticism. Chicago: Chicago University Press, 1994. 
DERRIDA, Jacques. Il monolinguismo dell'altro. Milano: Raffaello Cortina, 2004.

GOLDONI, Marco. Giustizia come distanza ed etica della traduzione. Aspetti della filosofia politica e giuridica di Paul Ricoeur. In (a cura di) Mattarelli S., Frontiere del repubblicanesimo. Milano: Franco Angeli, 2007.

ORTEGA Y GASSET, José. Miseria e splendore della traduzione. Genova: Il Melangolo, 2001. OST, François. Traduire. Défense et illustration du multilinguisme. Paris: Fayard, 2009.

PERNICE, Ingolf. La rete europea di costituzionalità, - Der Europäische Verfassungsverbund und die Netzwerktheorie, Zeitschrift für ausländisches öffentliches Recht und Völkerrecht Heidelberg Journal of International Law (HJIL), Band / v. 70, 2010.

POZZO, Barbara e Timoteo, Marina (a cura di). Europa e linguaggi giuridici. Milano: Giuffré, 2008.

RICOEUR, Paul. La traduzione. Una sfida etica. Brescia: Morcelliana, 2001.

SACCO, Rodolfo. Traduzione giuridica. Digesto delle Discipline Pubblicistiche. Torino: UTET, 2010.

STEINER, George. Dopo Babele: aspetti del linguaggio e della traduzione. Milano: Garzanti, 1994.

VESPAZIANI, Alberto. Diritti fondamentali europei. Casi e problemi di diritto costituzionale comparato. Torino: Giappichelli, 2009.

VESPAZIANI, Alberto. Comparison, Translation and the Making of a Common European Constitutional Culture. 9 German Law Journal, No. 5., 2008.

WEBER, Max. La scienza come professione. Milano: Rusconi, 1997. 